\title{
Miranda
}

Revue pluridisciplinaire du monde anglophone /

Multidisciplinary peer-reviewed journal on the English-

speaking world

1 | 2010

Variations on Darwin

\section{La composante darwinienne et la philosophie de Bergson dans la poésie de Thomas Hardy}

\author{
Ilaria Mallozi
}

\section{OpenEdition}

Journals

Édition électronique

URL : http://journals.openedition.org/miranda/702

DOI : $10.4000 /$ miranda.702

ISSN : 2108-6559

Éditeur

Université Toulouse - Jean Jaurès

\section{Référence électronique}

Ilaria Mallozi, «La composante darwinienne et la philosophie de Bergson dans la poésie de Thomas

Hardy », Miranda [En ligne], 1 | 2010, mis en ligne le 23 mars 2010, consulté le 16 février 2021. URL :

http://journals.openedition.org/miranda/702 ; DOI : https://doi.org/10.4000/miranda.702

Ce document a été généré automatiquement le 16 février 2021.

\section{cc) (i) () $\Theta$}

Miranda is licensed under a Creative Commons Attribution-NonCommercial-NoDerivatives 4.0 International License. 


\title{
La composante darwinienne et la philosophie de Bergson dans la poésie de Thomas Hardy
}

\author{
Ilaria Mallozi
}

1 Pour Delmore Schwartz, deux tendances opposées se croisent dans la poésie de Thomas Hardy. Intellectuellement, le poète anglais tend vers la science, mais il a également hérité d'un penchant substantiel pour la mouvance de la vie intérieure, comme si c'était pour lui une nécessité - « an undeniable necessity of seeing life from the inside of the human psyche » (Schwartz 126-7) - , nécessité qui fait de lui un pré-Moderniste. Or cette question est délicate, puisque l'alliance entre une démarche darwinienne et une démarche bergsonnienne parait contradictoire. Pourtant, si Hardy restait profondément sensible à la théorie de l'évolution, c'était aussi un lecteur du philosophe français, chez qui il espérait peut-être trouver une explication, non pas à la dynamique de l'évolution de l'espèce, mais aux élans spirituels et aux pulsions humaines plus inconscientes, afin de résoudre, pour le dire selon les termes de Hardy lui-même dans The Woodlanders, la tension entre trace primitive («primitive feelings») et la friction d'une sensibilité moderne (« modern nerves ») (Hardy 1978, 655).

2 La théorie de Bergson, comme l'explique Jacques Monod, dérive du Darwinisme, puisqu'elle analyse le principe téléonomique de la sélection en insistant sur le fait qu'elle n'opère qu'au sein de la matière vivante, de la biosphère. En découle une théorie de la vie conçue comme un élan, un courant, traversant la matière inanimée pour l'organiser. Ce «vitalisme métaphysique» (Monod 39) n'est cependant pas finaliste : l'homme n'a été ni cherché ni prévu par l'évolution, « [i]l en est plutôt la manifestation et la preuve de la totale liberté de l'élan créateur» (Monod 40). Pour Hardy, le retour vers le spiritualisme est bienvenu, même s'il s'agit d'un enthousiasme mesuré, comme en témoigne une lettre datée de 1925 où il avoue un manque d'engouement pour certaines conclusions de Bergson qui, selon lui, n'expliquent pas les dynamiques évolutionnistes, et visent au contraire à les rendre obscures :

You will see how much I want to be a Bergsonian (indeed I have for many years).

But I fear that his philosophy is, in the bulk, only our old friend Dualism in a new 
suit of clothes [...] You must not think me a hard-hearted rationalist for all this. Half my time (particularly when I write verse) I believe - in the modern use of the word - not only in things that Bergson does, but in spectres, mysterious voices, intuitions, omens, dreams, haunted places, etc., etc. But then, I do not believe in these in the old sense of belief any more for that; and in arguing against Bergsonism I have, of course, meant belief in its old sense when I aver myself incredulous. (Hardy 1965, 450-451)

3 Hardy se défie de l'intuition bergsonienne plaquée sur le discours rationnel, parce qu'elle implique un dualisme qui semble ramener vers une forme de foi sous un autre nom, sans pour autant rendre compte de toute cette spectralité si chère à Hardy - et la lettre révèle au passage ses propres contradictions (dont il a pleinement conscience) d'agnostique qui n'arrive pas à se défaire d'une forme de croyance en l'invisible. On pourrait objecter qu'une lettre, quelle que soit sa valeur autobiographique, ne constitue pas nécessairement un élément conceptuel fiable, au même titre qu'un essai ou une préface. Mais on ne peut que constater la correspondance qui existe entre l'œuvre romanesque et les écrits autobiographiques comme les lettres (à tel point que cette conformité semble même épuiser la question de ce qu'on pourrait appeler la censure autobiographique). Il est donc légitime de se pencher sur ces écrits autobiographiques, qui nous aident à explorer ce désir de déterminer la part du rationnel et de l'irrationnel chez l'individu. Hardy visait à démontrer l'existence d'une fracture perceptible chez tout être humain, qu'elle coupe les impressions des convictions, ou qu'elle scinde les sentiments qu'inspirent les moments mystiques, visionnaires, d'une forme de rigidité plus froide, gouvernée par la raison et un déterminisme plus perceptible lorsqu'une lumière crue vient accuser les traits. Ainsi, il décrit dans son autobiographie l'hiver comme une sorte de révélateur photographique faisant surgir les traits saillants de la personnalité à même la peau :

Cold weather brings out upon the faces of people the written marks of their habits, vices, passions, and memories, as warmth brings out on paper a writing in sympathetic ink. The drunkard looks still more a drunkard when the hectic blush becomes a stain now, the cadaverous complexion reveals the bone under, the quality of handsomeness is reduced to its lowest terms. (Hardy 1965, 177)

4 Le froid presque spectral donne à lire la personnalité, et ce palimpseste physiognomonique semble tenir à la fois de l'évolution darwinienne et d'une vision de la matière modelée par l'élan vital.

5 Ainsi, à lire ses observations autobiographiques, notamment à propos de l'exploration psychologique, il s'avère que Hardy nourrissait une profonde fascination pour la méthode philosophique de Bergson, même s'il a toujours privilégié l'observation scientifique de la realité. Et s'il éprouve, malgré la séduction indéniable des écrits de Bergson à l'époque (qui lui vaut d'ailleurs le Prix Nobel en 1927) la nécessité de prendre ses distances (comme en témoigne la lettre citée précédemment), c'est peut-être que l'apport que fait la philosophie de Bergson à la réception de la théorie de Charles Darwin pose effectivement problème. Vers la fin du dix-neuvième siècle, on associait l'évolutionnisme en Angleterre au "laisser-faire " capitaliste, à cause de la fameuse formule, «the survival of the fittest ». C'est Herbert Spencer qui avait délibérément forgé cette expression dans le contexte du débat évolutionniste, sans réfléchir aux implications éthiques de ce rapprochement, ce qui devait encourir la réprobation de Thomas Henry Huxley. Cette révolution scientifique - ou catastrophe, selon le point de vue à l'époque victorienne - qu'était l'évolution se vit ainsi attribuer une acception sociale, d'où la subséquente déformation que fit l'eugénisme de Francis Galton du 
darwinisme de Spencer. C'est donc une nouvelle torsion linguistique que Bergson fait subir à son tour au terme d'évolution dans ses ouvrages, in primis dans L'Evolution créatrice (1907), mais aussi dans ses essais sur le rêve, sur la perception du changement, sur l'énergie spirituelle et sur la mémoire. En instillant du spiritualisme dans les dynamiques évolutionnistes, Bergson impose une vision méditative de la vie sur terre. Bergson, selon James Rachels, est le philosophe qui avec le plus de force et de charisme, a réussi à transposer son besoin d'une nouvelle morale chrétienne sans nier les symptômes modernes de la dégradation religieuse, d'où ce modèle philosophique et littéraire qu'il a incarné, et qui a guidé une génération entière. Ainsi, Rachels cite une lettre de William James, dans laquelle le penseur américain magnifie son attachement aux idées du philosophe français : «O my Bergson you are a magician, and your book is a marvel in the history of philosophy» (Rachels 71). La version bergsonienne de l'évolution, si séduisante, semble en fait viser à cacher l'évidence d'un univers naturel autonome par rapport aux disciplines humaines, et exonéré de ses propres responsabilités. C'est bien ce que pressent Hardy en l'accusant de revenir au dualisme ("Our old friend Dualism in a new suit of clothes »), d'autant que cette tension entre intérieur et extérieur qu'explore Bergson se complique encore si l'on songe à la théorie de la sélection, qui présente une évolution humaine et animale qui se fait somme toute en harmonie avec le milieu et la réalité externe, argument que Darwin soutenait totalement, comme l'illustre cette citation qu'il fait de Wallace :

Wallace [...] argues that man, after he had partially acquired those intellectual and moral faculties which distinguish him from the lower animals, would have been but little liable to bodily modifications through natural selection or any other means. For man is enabled through his mental faculties : 'to keep with an unchanged body in harmony with the changing universe'. (Darwin 1980, 320)

6 En se plaçant à l'échelle de l'espèce, le Darwinisme ignore l'échelle de l'individu en souffrance, surtout quand il est voué à disparaître. Or, c'est cette dimension qui fascine Hardy. Il manque à la conception de Darwin la présence de la douleur et la perception de la souffrance humaine, comme Lennart Björk le souligne: "One aspect of the Darwinian impact on Hardy which has not received much attention is to be found in the ethical ramifications that Hardy saw in the theory of evolution » (Björk 337). C'est pourquoi Hardy s'interroge souvent, avec l'ironie qui lui est propre, sur l'humiliation qui est le lot de l'homme, reposant toujours et encore cette question de l'intentionnalité, dont il ne saurait se défaire tout en niant sans cesse la présence de Dieu, comme dans le poème «The Blinded Bird » par exemple : "all this indignity, / With God's consent » (Hardy 1976, 375). Dans un poème très connu, « Hap » (4), Hardy utilisait déjà pour Dieu l'adjectif «vengeful»; avec le temps, le poète va forger l'expression "Some Vast Imbecility " pour décrire dans "Nature's Questioning » la figure d'un dieu malade, idiot, écervelé (43) jusqu'à arriver à cet "Unsconscious Will » présent dans The Dynasts. De sorte que, dans la lettre où il soutient que la philosophie de Bergson n'explique pas entièrement les mystères de la vie dont lui-même est le témoin, le poète anglais veut se dissocier du Darwinisme social et du vitalisme métaphysique, pour remettre l'accent sur la question de la douleur : «If nature were creative she would have created painlessness, or be in process of creating it - pain being the first thing we instinctively fly from. If on the other hand we cannot introduce into life what is not already there, \& are bound to mere recombination of old materials, the persistence of pain is intelligible» (Hardy 1985, 84). La souffrance est le talon 
d'Achille de la philosophie de Bergson; cependant cette critique-là, Hardy aurait pu l'adresser à Darwin aussi, comme l'avait déjà fait George Eliot :

To me the Development Theory and all other explanations of processes by which things came to be, produce a feeble impression compared with the mystery that lies under the processes. (Eliot in Richardson, 85)

7 C'est sans doute dans le poème «The Lacking Sense » que Hardy répond à l'affirmation de Darwin présentant un être humain relativement stable dans un univers en mutation (« For man is enabled through his mental faculties : 'to keep with an unchanged body in harmony with the changing universe' »). Si tout le poème tourne autour d'une Nature qu'on découvre, tout simplement, aveugle, semblable à la téléonomie darwinienne, le poème attire l'attention sur la condition des êtres défectueux créés par les hasards du processus :

- 'And how explains thy Ancient Mind her crimes upon her creatures,

These fallings from her fair beginnings, woundings where she loves,

Into her would-be perfect motions, modes, effects, and features

Admitting cramps, black humours, wan decay, and baleful blights,

Distress into delights?' (Hardy 1976, 80)

8 On voit bien que ce qui fascine ici Hardy dans cette question qui problématise l'élan vital d'une Nature qui crée des spécimens humains défaillants, défectueux, c'est la mise en souffrance de l'individu. Hardy se sert donc surtout de l'évolution, qu'elle soit d'inspiration darwinienne ou réinterprétée par Bergson, comme d'une occasion de réviser quelques principes communément admis concernant l'éthique et l'idée de Nature (traditionnellement perçue comme une des incarnations de la Providence divine). Si Hardy sonde les principes scientifiques, c'est pour conceptualiser une vision de la vie sans Dieu, parce que la révolution scientifique et sociale impulsée par Darwin n'a été, en fin de compte, qu'une conjoncture parfaite pour interpréter la laïcité avec un autre langage. Le poète se tourne vers la science surtout parce qu'il y trouve des réponses tangibles aux problèmes d'intérêt collectif. La science satisfait sa soif de vérité, et il est proche en cela de Leslie Stephen : «dreams may be pleasanter for the moment than realities; but happiness must be won by adapting our lives to the realities " (Stephen in Cosslett, 15). En même temps, puisque les considerations agnostiques et scientifiques ne rendent pas compte de l'inaccessible espace de la douleur, il cherche à assouvir chez Bergson son désir d'aller au-delà du connu, vers ce que la littérature victorienne et fin de siècle recherchait encore.

Bergson décrit l'incapacité qu'a la science de sonder l'ineffable, puisqu'il faut intégrer le principe vital qui échappe pour lui à la loi de la pré-determination :

Le monde, laissé à lui-même, obéit à des lois fatales. Dans des conditions déterminées, la matière se comporte de façon déterminée ; rien de ce qu'elle fait n'est imprévisible: si notre science était complète et notre puissance de calculer infinie, nous saurions par avance tout ce qui se passera dans l'univers matériel inorganisé, dans sa masse et dans ses éléments, comme nous prévoyons une éclipse de soleil ou de lune. Bref, la matière est inertie, géométrie, nécessité. Mais avec la vie apparaît le mouvement imprévisible et libre. L'être vivant choisit ou tend à choisir. Son rôle est de créer. Dans un monde où tout le reste est déterminé, une zone d'indétermination l'environne. (Bergson 1959, 824)

Chez Hardy, la matière a aussi son intensité propre, ne serait-ce que parce qu'elle est soumise à la subjectivité humaine. En considérant des vers comme «shaped in my imagining / To the shade that a well-known head and shoulders / Threw there " extraits de « The Shadow in the Stone » (Hardy 1976, 483), nous nous apercevons qu'en 
poésie, Hardy (conscient ou non de ses affinités avec Bergson) visait à exprimer ce qu'il percevait comme le devenir de l'action dans la matière, semblable mutatis mutandis à cet au-delà de la matière comme "inertie, géométrie, nécessité » dont parle Bergson. Les vers de Hardy mêlent ici le phénomène de l'ombre portée et le fantasme du retour de la femme aimée; la matière se décline de façon imprévue, entre éclipse et apparition. Comme Schwartz l'a souligné, grâce à la poésie, Hardy opère ici un retour à l'intention inconsciente qui se matérialise dans l'objet même, alors qu'il s'agit d'un effet de mémoire. Tout comme la durée bergsonienne, le temps hardien est un temps qui ne peut donc pas être séparé de l'expérience humaine. Même le poème "Heredity ", qui fait du visage un palimpseste d'inspiration darwinienne, implique une expérience subjective plus bergsonienne, «that is I (Hardy 1976, 363). Le temps dont parle Hardy est le temps de la mémoire, qui vit dans l'espace des souvenirs, comme dans "Concerning Agnes » : " There she may rest like some vague goddess, shaped / As out of snow ». L'indétermination perdure, puisque le poème se conclut sur «I cannot tell! » ( 862). Dans le poème "Wessex Heights ", le poète confesse son appartenance aux lieux :

There are some heights in Wessex, shaped as if by a kindly hand For thinking, dreaming, dying on, and at crises when I stand Say, on Ingpen Beacon eastward, or on Wylls-Neck westwardly

I seem where I was before my birth, and after death may be. (Hardy 1976, 319)

11 Pour Hardy l'espace est supérieur au temps, parce que dans les lieux, présent et passé co-existent, comme chez Bergson : «cette alliance du présent et du passé est féconde dans tous le domaines» (Bergson 1911, 3). Cette continuité que Hardy cherchait désespérément entre présent et passé, il la trouve dans les paysages, dans les formes métriques, autant que dans les traits dominants de la psychologie humaine. Il est en cela plus proche de Bergson que de l'accent darwinien sur une nature animale et végétale impassible et cruelle: Bergson ne proposait-il pas une idée du présent et du passé «où rien ne serait sacrifié de ce que nous présentent nos sens et notre conscience » (Bergson 1911, 8-9)?

12 Cette inaptitude à sacrifier le passé peut expliquer l'attitude ambiguë envers une religion à laquelle Hardy ne croit pas, tout en continuant à en préserver la trace en la liant à la poésie, comme dans Late Lyrics (1922). Le poète y semble conscient de vivre un moment historique unique, et ses mots nous montrent son appartenance à une humanité très partagée, mais il reste attaché à l'idée d'église, proposant un idéal d'éthique fondée sur la laïcité, qu'il veut concilier avec le legs de la science :

In every poetry, pure literature in general, religion - I include religion, in its essential and undogmatic sense, because poetry and religion touch each other, or rather modulate into each other; are, indeed, often but different names for the same thing - these, I say, the visible signs of mental and emotional life, must like all other things keep moving, becoming; even though at present, when belief in witches of Endor is displacing the Darwinian theory and "the truth that shall make you free", men's minds appear, [...], to be moving backwards rather than on. [...] one may ask, what other purely English establishment than the Church, of sufficient dignity and footing, with such strength of old association, such scope for transmutability, such architectural spell, is left in this country to keep the shreds of morality together? (Hardy 1976, 448-9)

13 Certes, Darwin a balayé les conceptions religieuses obsolètes de la création de Dieu, mais Hardy rêve d'une église où la moralité ne serait donc plus liée à la foi, qui réinventerait entièrement sa doctrine, un peu sur le mode de l'élan vital bergsonien, et 
qui pourrait s'approcher de la science; dans ce cas-là il est possible aussi de penser au dessein naturel de l'évolution et à la logique du progrès humain en termes d'instinct et de raison.

La ferveur laïque qui animait la recherche religieuse de Hardy est bien décrite dans l'une de ses lettres adressées à E. Clodd à l'occasion de la publication de son livre, Thomas Henry Huxley, en 1902 :

If the doctrines of the supernatural were quietly abandoned to-morrow by the Church, \& 'reverence \& love for an ethical ideal' alone retained, not one in ten thousand would object to the readjustment, while the enormous bulk of thinkers excluded by the old teaching would be brought into the fold, \& our venerable old churches \& cathedrals would become the centres of emotional life that they once were.

Well : what we gain from science is, after all, sadness, [...] that the world exists is a fact absolutely logicless \& senseless. (Hardy 1982, 5)

Ainsi, le sacré lavé de la doctrine chrétienne, réinventé sous une forme d'éthique pure, liée à l'église comme communauté et bâtiment de pierre, pourrait compléter le " gain " offert par la science, cette lucidité qui a privé le monde de sa téléologie. Ainsi, le poème « New Year's Eve » (Hardy 1976, 231) ouvre une fenêtre dialogique, où l'homme encore une fois scrute la noluntas de Dieu, comme le souligne l'adjectif composé allitératif "Sense-sealed ", suggérant une dynamique mystérieusement aveugle à tout dessein, l'équivalent de la téléonomie darwinienne :

Then he : « My labours-logicless-

You may explain; not I :

Sense-sealed I have wrought, without a guess

That I evolved a Consciousness

To ask for reasons why ».

La dernière strophe montre le rapport amoral entre l'humanité et un «Dieu » qui n'est plus chrétien, créant par cœur ( in his unweeting way ») avec une énergie extatique, sans se soucier le moins du monde de la bizarre créature qui voudrait lui demander des comptes, l'homme :

He sank to raptness as of yore,

And opening New Year's Day

Wove it by rote as theretofore,

And went on working evermore

In his unweeting way.

17 Le scénario du poème "The Lacking Sense » est donc présent à nouveau dans "New Year's Eve ", reposant la question de la souffrance et de la conscience humaine dans un monde aveugle où l'émotion n'est plus pertinente, comme l'indique l'autobiographie: "The emotions have no place in a world of defect, and it is a cruel injustice that they should have developed in it " (Hardy 1965, 149). Dans une lettre adressée à Clodd, Hardy écrit de même, toujours à propos de " New Year's Eve » :

Many thanks for your letter about my New Year's fantasy or dream in the F. R.

[Fortnightly Review]. [...] As you say, people will no doubt mistake it for a belief. It is Feuerbach who says that God is the product of man. [...] On the other hand I quite enter into Spencer's feeling - that it is paralysing to think what if, of all that is so incomprehensible to us (the Universe) there exists no comprehension anywhere. (Hardy 1982, 244)

Se plaçant dans la filiation de Spencer, Hardy exprime ici son impuissance face à l'absence de compréhension de notre univers ; il revient sur sa crainte existentielle face 
au hasard. Hardy combine deux fortes pulsions: le désir intellectuel d'adhérer aux vérités scientifiques liées au Darwinisme, mais en même temps, il ne peut combler le manque provoqué par l'anéantissement de la croyance religieuse. Ainsi, nous avons vu qu'il souhaite croire que le bâtiment de l'église, même débarrassé de ses fausses croyances, puisse garder quelque force de cohésion face aux mutations qui ébranlent le statut de l'homme. De même, dans le poème "God's Funeral »(Hardy 1976, 267), en voyant passer le cercueil du défunt Dieu, anéanti par la science, le locuteur s'adresse aux croyants (comme pour participer au sentiment de souffrance et de perte), mais ne peut s'empêcher de jouer en partie le rôle du croyant, attentif à quelque lumière qui pourrait poindre à nouveau. Le poète spéculait sur l'idée d'une religion fondée sur l'éthique et en même temps, bien enracinée dans l'histoire des lieux et des gens. Patrick Yarker met l'accent sur la churchiness de Hardy, son habitude d'aller à l'église pour chercher la compagnie des petites gens. Le poète connaissait bien les changements de la société, mais ne voulait pas s'éloigner de la vie rurale qu'il avait menée jusqu'à la fin de son existence (les traditions, les mythes, etc...), en un mot, le Wessex ancestral, qui semble plonger ses racines jusque dans la préhistoire :

He had an instinctive feel for the immemorial way of life, regulated by the seasons, punctuated by secular or ecclesiastical festivals, and maintained by 'practices which had suffered no mutilation at the hands of time'. Moreover, in those ancient regions evidence is plentiful of occupation and husbandry since prehistoric times. Hardy's sense of the past was fostered by the propinquity of these earlier inhabitants, who has never really vanished from the scene. (Yarker, 317-8)

19 Il est évident que, pour Hardy, Darwin n'avait fait que réveiller, et non pas provoquer, le conflit entre la croyance et la raison. Dès le poème « To Outer Nature » (Hardy 1976, 37), une de ses premières compositions, Hardy annonce sa difficulté à accepter la disparition de l'osmose avec la Nature, dont il a la nostalgie; or la maturité intellectuelle interdit cette naïveté, opposant en particulier le passage sur l'enfance à la Wordsworth à l'imagination darwinienne :

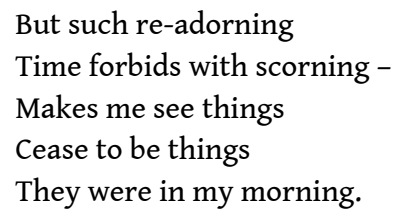

Walter F. Cannon montre comment la poésie a dû changer, et changer ses métaphores aussi, pour ne pas sembler ridicule et inutile (Cannon 166). L'éternité que Keats pouvait donner à son rossignol devenait, après Darwin, simplement risible, presque naïve. L'amour ne pouvant plus être vécu à la manière romantique, la religion avait aussi besoin d'être sauvée de ses idéaux irréalisables de pureté. Voilà le sens de la modulation entre la poésie et la religion que Hardy conseille dans son "Apology ", puisque la poésie se substitue, en quelque sorte, au sacré, « because poetry and religion touch each other, or rather modulate into each other ; are, indeed, often but different names for the same thing ".

21 La philosophie de Bergson a sans doute aidé Hardy à imaginer une façon de raisonner capable de concilier les contradictions et de réaliser la fusion des contraires («the fusion of that which logic divides » Taylor, 140). Les mots de Bergson représentent une manière de faire confiance à l'homme et au monde. L'incipit de "Afterwards" démontre la même tentative en poésie, quand le locuteur joue à l'observateur détaché en allant jusqu'à imaginer sa propre mort, déclinant le paradigme des éléments favoris 
de la nature - d'une feuille verte ou d'un hérisson aux étoiles - mais perçus par d'autres, et cristallisant le souvenir de celui qui les aimait, en une sorte de continuité par le biais de la communauté :

When the Present has latched its postern behind my tremulous stay,

And the May month flaps its glad green leaves like wings,

Delicate-filmed as new spun silk, will the neighbours say,

'He was a man who used to notice such things'? (Hardy 1976, 511)

«Afterwards» joue sur deux plans spatiaux-temporels juxtaposés: le temps coule régulièrement, mais n'est pas accessible à la conscience, dans la mesure où le temps mental (y compris la mémoire) touche souvent les zones obscures de notre cerveau. On touche ici à ce qui rapproche peut-être le plus Hardy de Bergson, la fascination pour la mémoire, véritable hantise chez Hardy, objet d'étude pour Bergson. Quand l'esprit explore les souvenirs, le risque est grand de laisser la conscience à distance, ce qui permet de reposer les questions de la nature et de la trace, de l'esprit et de la matière, fût-elle biologique. Bergson tisse un lien entre matière, conscience et liberté, pour conceptualiser la mémoire :

Conscience et matérialité se présentent donc comme des formes d'existence radicalement différentes, et même antagonistes, qui adoptent un modus vivendi et s'arrangent tant bien que mal entre elles. La matière est nécessité, la conscience est liberté ; mais elles ont beau s'opposer l'une à l'autre, la vie trouve moyen de les réconcilier. C'est que la vie est précisément la liberté s'insérant dans la nécessité et la tournant à son profit. Elle serait impossible, si le déterminisme auquel la matière obéit ne pouvait se relâcher de sa rigueur. Mais supposez qu'à certains moments, en certains points, la matière offre une certaine élasticité, là s'installera la conscience. (Bergson 1959, 824, c'est moi qui souligne)

Bergson établit un espace de relation entre conscience et matière, pour démontrer que l'homme a besoin de ces pôles opposés pour vivre, penser, et être libre. Il ne réconcilie pas les conflits, il les enchâsse en articulant matière et conscience. Pour Hardy, ce conflit se joue non seulement entre esprit et matière, mais aussi entre poésie (matière des mots) et les «impressions et convictions » de la conscience. Ainsi, la conscience crée l'expérience poétique mais en dérive aussi, et diffère des données matérielles et incontestables de l'existence, dont la mort est l'inéluctable finalité. Hardy rejoint Bergson sur la présence continue, dynamique de la mémoire : comme l'a dit Bergson dans Matière et Mémoire, « il ne peut y avoir dans le cerveau une région où les souvenirs se figent et s'accumulent. La prétendue destruction des souvenirs par les lésions cérébrales n'est qu'une interruption du progrès continu par lequel le souvenir s'actualise " (Bergson 1959, 270). Mémoire et souvenir ne relèvent pas de l'instant présent, mais d'une concentration subjective des données de notre conscience, qui nous donne l'impression d'une continuité vitale entre passé et présent.

Dans "Afterwards", dernier poème de Moments of Vision, c'est l'ironie qui, comme toujours, sauve le poète de sa propre conscience, pour lui permettre de vivre au-delà de toute conception prédéterminée de la mémoire. On voit donc que si les approches ont des affinités, les conclusions divergent; Hardy reste dans le doute et une forme de nostalgie, comme s'il ne trouvait pas en Bergson la lumière capable d'éclairer la question de la détresse, alors que Bergson peut accepter l'aveuglement de l'instinct, comme le rappelle Dennis Taylor :

The question Bergson pursues is how to turn the intellect around, how to reconcile intellect and its belated abstractions with instinct which is blind but is moulded on life. (Taylor 140-1) 


\section{BIBLIOGRAPHIE}

Bergson, Henri. La Perception du Changement. Oxford : Clarendon Press, 1911.

--- Euvres. Ed. A. Robinet et al. Paris : PUF, 1959.

Bullen, J. Barrie. The Expressive Eye. Oxford : Clarendon Press, 1986.

Cannon, W. F. « Darwin's Vision in On the Origin of the Species ", in The Art of Victorian Prose. Ed. G. Levine at al. New York : Oxford University Press, 1968, 154-176.

Cosslett, Tess. «The Scientific Movement » and Victorian Literature. Sussex : The Harvester Press ; New York : St. Martin's Press, 1982.

Darwin, Charles. The Descent of Man and Selection in Relation to Sex. 1871. Chicago : Chicago

University Press, 1980.

Hardy, Florence Emily. The Life of Thomas Hardy : 1840-1928. London : Macmillan, 1965.

Hardy, Thomas. Collected Poems. 1930. Ed. James Gibson. London : Macmillan, 1976.

---. The Woodlanders. 1887. London : Macmillan, 1978.

---. The Collected Letters of Thomas Hardy (Vol. Three 1902-1908). Ed. Purdy, R. L. et al. Oxford :

Clarendon Press, 1982. 
---. The Collected Letters of Thomas Hardy (Vol. Five 1914-1919). Ed. Purdy, R. L. et al. Oxford :

Clarendon Press, 1982. 1985.

---. The Literary Notes of Thomas Hardy (vol.1). Ed. Björk, Lennart. London : Macmillan, 1985.

Monod, Jacques. Le Hasard et la nécessité : essai sur la philosophie naturelle de la biologie moderne. Paris : Seuil, 1970.

Rachels, James. Created from animals : the moral implications of Darwinism. Oxford : Oxford University Press, 1990.

Richardson, Angelique. Love and Eugenics in the Late Nineteenth Century. Oxford : Oxford University Press, 2003.

Schwartz, Delmore. « Poetry and Belief in Thomas Hardy » . In Hardy : A Collection of Critical Essays. Ed. Guérard, A. J. Englewood Cliffs (NJ) : Prentice-Hall, 1963, 123-134.

Taylor, Dennis. Hardy's Poetry 1860-1928. London : Macmillan, 1981.

Yarker, Patrick M. « Later Victorian Novelists. Meredith, Hardy and Gissing ». In Sphere History of Literature. The Victorians. Ed. Pollard, A. London : Sphere, 1988, 307-360.

Zietlow, Paul. Moments of Vision. The Poetry of Thomas Hardy, Cambridge (Mass.) : Harvard University Press, 1974.

\section{NOTES}

1. Voir à ce propos la lettre à Clodd dans laquelle Hardy écrit: "That theory of consciousness in plants is an arresting one: but I have always known it intuitively, \& hate maiming trees in that account » (Hardy 1982, 331).

\section{RÉSUMÉS}

Les théories de Darwin ont influencé à la fois le poète et romancier Thomas Hardy et le philosophe Henri Bergson, l'un étant profondément marqué par les mutations scientifiques qui sapent les bases de la religion, l'autre s'inspirant du darwinisme pour le modifier profondément en introduisant un élan vital qui réintroduit une forme de métaphysique. Cet article analyse les affinités inattendues qui existent entre la pensée de Bergson et celle de Hardy, permettant de nuancer la perception du legs de Darwin. Bien que le poète ait été fasciné par Bergson (il confie dans une lettre son désir de céder à la séduction de son œuvre) il reconnaît en fin de compte que le «dualisme» de Bergson ne saurait correpondre à l'éclatement du monde post-darwinien. Darwin et Bergson ont ainsi permis à Hardy de mieux articuler sa vision de la perte toujours problématique de la religion anéantie par les révélations de la science, et inspiré ses poèmes les plus empreints de compassion, même si la théorie de Darwin et la pensée de Bergson fonctionnent en fin de compte selon des modes contradictoires.

This article addresses the affinities between Henri Bergson's philosophy and Thomas Hardy's poetry. Both draw upon Charles Darwin's theories of natural science: Hardy was greatly 
influenced by them, whereas Bergson transmuted Darwinism as his élan vital stands in deliberate contrast with Darwin's materialism. Although the French philosopher fascinated the poet (in a letter to Saleeby, he wrote : "You will see how much I want to be a Bergsonian »), he recognized that Bergson's «dualism» was totally incongruous with the incorrigibly plural post-Darwin world that Hardy's Weltanschauung embraced. Both Darwin and Bergson helped Hardy articulate his complex response to the loss of religion, and influenced some of his most compassionate, spiritual poems, despite the fact that their theories worked in different ways.

\section{INDEX}

Mots-clés : conscience, élan vital, évolution, hasard, matière, mémoire, nature, poésie, religion, souffrance

Keywords : chance, conscience, evolution, vital impetus, matter, memory, nature, pain, poetry, religion

\section{AUTEURS}

ILARIA MALLOZI

Ministère italien du patrimoine culturel

ilaria.mallozzi@gmail.com 